\title{
ON A FORM
}

op

\section{ALCOHOLIC SPINAI PARALYSIS.}

\author{
BY \\ W. H. BROADBENT, M.D., F.R.C.P. \\ LECTURER ON MEDICINE AT, AND PHYSICLAN TO, ST. MARY's HOSPITAL.
}

(Received December 11th, 1883-Read February 12th, 1884.)

Sefteral cases of a remarkable form of spinal paralysis have come under my notice at different times, but, with the exception of the first, which occurred in St. Mary's Hospital when I was resident medical officer there, the patients have been seen in consultation once only, and no post-mortem examination has been obtained, so that it has been impossible to give anything like a complete and connected account of the disease. This I am now able to do through the zealous co-operation of $\mathrm{Dr}$. H. F. E. Harrison, of Shepherd's Bush Green, who called me to a case of the kind on the 3rd of April, 1883.

The patient was a gentleman, æt. 42. He was by profession a barrister, but had never practised, having independent means. As a young man he was a cricketer, and had played for his college and in the eleven of his county, but he early developed a liking for wine and gradually became inert and sedentary. In March, 1875, he came under Dr. Harrison's care and was then a confirmed dipso- 
maniac. His favourite drink was port, and his habit was to begin drinking about mid-day and to go on more or less all day and through the night. He spent most of his time in bed, reading and drinking, and of late years was seldom seen out of his house except at the University cricket match or on some similar occasion.

It is probable that these habits had an important influence on the form taken by the injurious effects of alcohol. He never had pronounced delirium tremens, but at times complained of wakeful nights and of seeing spectres, and he was easily startled and frightened. For some months his friends had observed a degree of failure of mental power, shown chiefly by his tendency to ask the same question or repeat the same remark over and over again.

The precise date of the access of the paralysis cannot be fixed. He had been suffering much from vomiting and had been in bed for a month; slight weakness of the arms had been noticed for about a fortnight before April 3rd, when I was called in consultation, but it had only interfered with the use of the hands in holding his paper or carrying his glass to his mouth for a day or two.

On examination at this time (April 3rd), the attention was at once called to the hands, which could no longer hold an object, and there was conspicuous dropping of the wrist, especially of the right limb. When the forearm was prone the hand fell by its own weight, and extension was impossible ; the grasp also was extremely feeble, partly from loss of opposition to flexion at the wrist by the paralysis of the extensors, but also from weakness of the flexors. Pronation and supination could be effected in a languid way ; flexion of the elbow was fairly energetic, and the hand could be carried promptly to the head and behind the neck, although of course it hung loosely at the wrist. The left upper extremity was in a similar condition, but the paralysis of the extensors of the wrist had not gone so far, and the hand could be brought into line with the forearm, although, in the absence of effort, it dropped like the right.

The muscles of the forearm were of average volume, but 
soft to the feel; they were very tender when handled. The hands were pale and slightly puffy with a purplish tinge.

The legs were very thin as compared with the arms. They could be raised from the bed, and flexion and extension of all the joints could be performed readily. The patient, however, could not stand alone.

The knee-jerk was entirely absent.

In raising himself into the sitting posture the patient required assistance, and it was evident that the muscles of the trunk were enfeebled. He could sit upright, how. ever, and turn in bed.

As the legs hung down the feet could be seen to swell sc that in a few minutes they became distinctly puffy.

There was no paralysis of the face or tongue, and the voice was strong and distinct.

Sensation was apparently unaffected. There was no umbilical reflex, but when the feet were tickled they were quickly withdrawn, and the patient exclaimed that he could not bear it.

The cheeks had a good colour, which, although due to dilated capillaries, gave the patient the appearance of health. The eyes were unduly open, giving him a startled or wild look and the pupils were large, but equal and acting well.

The pulse was regular, but frequent, small, short, and weak ; there was no distinct cardiac impulse or apex beat; the first sound was short and fairly loud, the second extremely weak and following the first at an abnormally brief interval. The general effect was a singular accentuation of the first sound, the second following it immediately like a weak echo.

The liver was large, and its margin was felt to be firm and thick; the abdomen presented nothing remarkable.

The urine was of a dark-red (almost brick-red) colour ; acid in reaction, with a sp. gr. of 1028, throwing down a copious deposit of red urates ; it contained neither albumen nor sugar. 
All alcohol was withdrawn; beef-tea and milk were given as food, and Liq. Strychniæ mvj was ordered as medicine.

He became rapidly worse, and on April 9th he lay on his back unable to turn in bed or move his body in any way; his arms were lying along the body, he could just raise the right hand from the bed and drag it partly on to the side of the abdomen; the left he could only move slightly but could not lift, being thus more affected than the right. The hands fell by their weight into extension or flexion according to the position of the forearm. From their position, which was somewhat dependent, they were much more puffed and swollen than before, and pink in colour.

The legs could only be freely drawn up and could not be lifted from the bed. There was no knee-jerk or ankle-clonus. Tickling the soles made him cry out and withdraw the feet.

There was no umbilical reflex, and tickling of the flank excited no complaint.

The aspect and expression of the face were more natural than at the previous consultation and the pupils less dilated, perhaps because of the withdrawal of alcohol. The voice was good and articulation quite distinct.

With the general increase of paralysis there was now obvious paralysis of the diaphragm. The patient did not complain of respiratory distress, but the movements of the upper part of the chest were observed to be greatly exaggerated, and both inspiration and expiration were noisy. On examination it was seen that the abdominal respiratory movements were reversed, the epigastrium receded in inspiration and was forced outwards in expiration, showing that the diaphragm did not contract but yielded passively to pressure. At this time, as has been already stated, the voice was still strong and speech unaffected in any way, but in the evening after a sleep of some duration this was no longer the case; it could scarcely be made out what he said. This was at first supposed to be due to dryness of the mouth or to the patient being only half awake, but it was soon seen to be an effect of paralysis. 
Next day, April 10th, the affection of the speech was the first symptom to claim attention. It was such that without some clue to what the patient said it was impossible to understand him, but short answers to questions could be followed. There was no aphasia. He had words at command and could say them after a fashion; again there was no obvious paralysis of the lips or tongue. The lips moved in speech and he was able to drink, a little flaid escaping, but not more than might be expected in the helpless condition of the patient; the tongue was protruded at command and carried readily from side to side; the voice, moreover, was fairly loud and clear. The indistinctness of articulation was obviously due partly to want of breath, partly to want of power to maintain the vocal cords in apposition for phonation; a phrase was cut up into very short fragments each of which required a special effort, and only the beginning of individual words was actually pronounced.

The paralysis of the respiratory muscles had made rapid progress. When the trunk was exposed the alternating action of the chest and abdomen was now extremely conspicuous ; as the upper part of the thorax rose the abdomen fell in, and as the thorax fell the abdomen rose, these movements being sudden and extensive; the diaphragm was simply flapping to and fro. The lower ribs also, which on the previous day had expanded as well as risen, were now drawn in during inspiration. Of course under these circumstances there was very little entry of air into the lower part of the lungs, and the respiratory murmur was scarcely audible.

There was no very marked aggravation of the paralysis of the limbs; both arms and legs could still be moved slightly, the legs better than the arms. Tickling of the soles excited movements and exclamations, and sensation was apparently unaffected.

The hands were scarcely as much swollen as on the previous day; the feet not at all. The extremities were warm. 
The urine was passed naturally.

The expression of the face was natural and the colour good.

The pulse had the same character as before; short, weak, soft, and small. The first sound of the heart was short, sharp, and fairly loud, the second extremely feeble over both aorta and pulmonary artery.

Later in the day he slept, and as before was much worse on waking; the speech more indistinct and the paralysis of the respiratory muscles aggravated. Râles began to be heard in the bronchi and trachea. From this time there was a steady advance of the symptoms, and death took place about noon on April 11th.

Permission to examine the spinal cord was obtained, and this was done at 9 a.m. on the 12 th, twenty-nine hours after death, before the body was cold.

The thin legs contrasted with the somewhat bulky body and well-developed arms, and the experienced assistant spontaneously called attention to the extraordinary whiteness and the swollen condition of the hands, and to the wet and sodden state of the structures of the back.

When the spinal canal was opened there was nothing noticeable outside the sheath of the cord, and the dura mater was quite normal. The cord itself, again, when exposed was firm to the touch, and the only abnormal appearance was its whiteness and the small size of the vessels on its surface.

The most careful microscopic examination of stained and hardened specimens failed to detect any structural change in the cells or fibres.

The first case of the kind which came under my observation was in St. Mary's Hospital' while I was resident medical officer, but I have been unable to find my notes of it. The patient was a young woman who had taken to drinking after being deserted. Other cases more or less similar seen at different times may be briefly mentioned.

One was that of a young married lady seen with Dr.Myrtle, of Harrogate, in April, 1882, and described by him at the 
meeting of the British Medical Association in August of that year. The lower extremities had been slowly losing power but could still support the weight of the body ; the extensors of the wrist and hand were beginning to show weakness, but the most striking and characteristic feature was the complete loss of tone in the capillaries, so that when the feet were allowed to hang down they could be seen to fill with blood and become turgid and swollen.

A widow lady, æt. about 50, long addicted to alcohol and who for two or three years after the death of her husband had indulged in brandy unrestrainedly, was seen in consultation with the late Dr. Rhodes, of Weymouth. She was in bed, but still able to use her legs and support her weight upon them, while the hands had become useless, and, as she held them out, dropped at the wrist and could not be extended. They were pale and puffy, the dorsum being swollen and rounded as in dropsy. The muscles generally were extremely soft and tender. There was no disease of heart or kidneys. This patient died about a fortnight later from gradual paralysis of the respiratory muscles apparently beginning with the diaphragm.

A case seen with Mr. G. Amos Duke was at a more advanced stage. The patient, æt. 31 , was the wife of an innkeeper who had for two or three years taken at least a bottle of brandy per day. A child to which she had given birth seven years before was said to have manifested syphilis, and she had had repeated miscarriages since.

For three months her legs had dragged, and three weeks before our consultation she was said to have had sudden loss of power in all her limbs, with speechlessness and difficulty of swallowing. She was, however, away from home at the time, and not under observation, and had travelled from Eastbourne to London afterwards, without difficulty. For a fortnight paralysis had gradually been coming on, and when seen on November 9th, 1878, there was marked loss of power in the upper extremities generally, with dropping of the wrists, great impairment of power also in the lower limbs, without 
absolute paralysis, while the diaphragm was apparently completely paralyzed, respiration being thoracic only. There was aphonia, partly from want of breath, but also from some degree of laryngeal paralysis; liquids entered the larynx and provoked choking and cough. The movements of the soft palate were natural, and the tongue was well protruded. She died shortly afterwards from asphyxia.

In the case just spoken of there was a suspicion of syphilis, and another patient seen January 1st, 1884, with Mr. Wheeler, near Kew, was actually under treatment for this disease, which he had contracted in March or April, 1883. He was thirty-nine years of age, but looked forty-nine at least, and had been a heavy drinker from the age of twenty, taking at times extraordinary quantities of spirits. At the end of October, when he came under Mr. Wheeler's care, his legs were weak and shook under him as he walked. This had been noticed for a fortnight, and when he sat down he had needed helping up. About the middle of November he became unable to walk, and first the left and then the right hand became paralyzed, the paralysis beginning at the ulnar side. When I saw him on January 1st he lay helpless in bed; the legs could be feebly drawn up, but could not be put down again without assistance, the upper extremities were helpless but not immoveable, there was slight paralysis about the mouth, and the diaphragm was paralysed. Sharp pains shot down the legs from time to time, and there was incontinence of urine, these latter being exceptional features. The hands and feet had the pale puffy appearance mentioned in other cases, and the muscles were soft and flabby.

The paralysis invaded the walls of the chest, and the patient died on January 8th.

In trying to assign to this form of paralysis its proper place among the diseases of the nervous system, it will be well to recapitulate briefly its characters and peculiarities. 
There is first gradually increasing weakness of the lower extremities which may be noted for some time, when marked loss of power becomes manifest in the extensor muscles of the forearm giving rise to double drop-wrist. It cannot be said that when this is first apparent there is no distinct paralysis elsewhere; the lower extremities and the back are weak, but the drop-wrist may be complete, and the flexors of the hand may also be paralysed so that the hand is like a flail, while the movements of the elbow and shoulder are good and fairly vigorous, and the patient can support himself on his feet. As the paralysis advances all the muscles of both upper and lower extremities are affected, and the limbs can only just be moved, the arms being as a rule more seriously implicated than the legs; the muscles of the trunk also are paralyzed so that the patient cannot raise himself or turn in bed. The sphincters retain their functional power, and sensation is not affected. There is no pain from first to last, though the muscles may be tender on handling. An exception to these statements, however, is furnished by the case last seen. No convulsion or jactitation occurs.

Death is caused eventually by paralysis of the respiratory muscles, and the diaphragm is the first of these to be affected. Instead of descendingduring inspiration it remains flaccid and is forced upwards by atmospheric pressure on the abdomen ; in expiration the reverse of this takes place, so that the chest and abdomen no longer act together in respiration, rising and falling simultaneously, but their movements alternate. As the chest expands the abdomen falls in, and as the chest contracts the abdomen is protruded. The thoracic movements proper are the more energetic in order to compensate for the loss of the diaphragmatic respiration, so that the see-saw action of the chest and abdomen is very conspicuous, and at first there is actual expansion of the lower segment of the chest. Later the muscles of the chest become affected, apparently from below upwards, when, of course, death ensues from apnoa. 
A striking feature of this form of paralysis is the loss of tone in the capillaries. It has been stated in the description of the case (p. 135) that when the patient was seated on the edge of the bed with the legs hanging down, the feet were filled with blood and could literally be seen to swell and become puffy. This was equally conspicuous in Dr. Myrtle's case, the lividity being more marked. The capillaries yield and permit themselves to be distended. The pale or livid and swollen condition of the hands, when they could no longer be raised but lay in the bed by the side of the patient, was due to the same cause, as was also the unusual degree of saturation of the structures of the back with moisture found after death. It is the degree of capillary paralysis which is remarkable, and the early period at which it is observed; almost all paralyzed limbs swell when allowed to remain long in a dependent position, but in no other form of paralysis is the stagnation of blood so complete and immediate or the swelling so rapid, and it is to be noted that this is observed when the loss of power is not very considerable.

There can be no question as to the seat of the disease giving rise to the symptoms described; this is obviously the spinal cord. As to its nature no discussion is required to exclude myelitis, diffuse or localised. Very brief consideration again is sufficient to show that the affection is not so-called acute anterior poliomyelitis occurring in adults; the regressive paralysis of Dr. Barlow, which is never fatal by paralysis of the respiratory muscles; it is strikingly progressive instead of being regressive. The disease which it most resembles is acute ascending paralysis, and it is probable that the condition of the cord is essentially similar, no lesions being discoverable by any mode of preparation at present known. The features common to the two are the progressive advance of the paralysis till death is brought about by paralysis of the respiratory muscles; the absence of fever or pain or spasm; the fact that sensation is not affected and that the sphincters retain their functional activity. The 
principal difference is the order in which the muscles are invaded.

In acute ascending paralysis the legs are first affected and the loss of power gradually extends from below upwards; this becomes particularly noticeable when the chest is reached; the intercostals are first attacked and respiration is carried on by the diaphragm after the thorax has become motionless. The only case of acute ascending paralysis which I have seen presented a very different clinical picture when the stage of dyspncea was reached to that observed in these cases. The patient could not lie down, but insisted on being supported in the sitting posture with the arms well raised by attendants so as to draw up the chest, while the head, which the neck was no longer able to support, had to be steadied and prevented from falling forwards or to one side ; another difference appears to be that acute ascending paralysis is more common in men than in women. The cases collected by $\mathrm{Erb}^{1}$ are twelve men and four women, while alcoholic spinal paralysis is more frequently seen in women. I find no note by observers of loss of tone in the capillaries in acute ascending paralysis, and I did not remark it in the case I first saw.

Admitting the similarity between the condition under consideration and acute ascending paralysis, I think the difference sufficiently marked and constant to warrant a distinction by means of a name. This name I should take from the etiology. In all the cases which have come under my observation there has been excessive indulgence in alcohol, and $I$ have no hesitation in assigning this as the cause of the disease, the alcohol exerting an influence on the grey matter of the spinal cord probably similar to that which in the brain gives rise to delirium tremens.

A point worthy of note is that in all the cases except that which forms the subject of this paper and that seen since it was written the patients were women. Dr.

1 'Ziemssen's Handb. der speciellen Pathologie und Therapie,' Bd. xi, 2te Hälfte, 2te Abth., S. 324 (Trauslation, vol. xiii, p. 735), 
Rhodes's and Dr. Myrtle's patients were ladies in good position ; Mr. Duke's the wife of a publican ; the hospital patient was the discarded mistress of a rich man. It is not probable, however, that sex is the factor which determines the direction taken by the injurious effects of alcohol. The affection of the spinal cord does not apparently occur among the women who are admitted into hospitals, or it would have been frequently observed and described; that is, it does not occur in women who have to work for their living or who have laborious domestic duties. It would appear that unrestricted access to stimulants throughout the entire day, and an indoor inactive mode of life, were the conditions which together invited the disease ; these were just the conditions common to the five women and two men who have come under my observation. 